\title{
CONFORMATION PROPERTIES OF ONE ISOLATED POLYELECTROLYTE CHAIN IN D DIMENSIONS
}

\author{
P. PFEUTY
}

Laboratoire de Physique des Solides (*), Université Paris-Sud, Centre d'Orsay, 91405 Orsay Cedex, France

\section{R. M. VELASCO and P. G. DE GENNES}

Laboratoire de la Matière Condensée, Collège de France, place Marcelin-Berthelot, 75231 Paris Cedex 05, France

(Reçu le 4 octobre 1976, accepté le 16 novembre 1976)

\begin{abstract}
Résumé. - Les exposants $\gamma$ et $v$ décrivant les propriétés asymptotiques d'un polyion en solution dans la limite des grands poids moléculaires sont déterminés à partir d'une théorie Lagrangienne à zéro composante près de $d=6$ dimensions. La taille moyenne du polyion $R$ varie comme $N^{2 / d-2}$ où $N$ est le nombre de monomères. Ce résultat $v=2 / d-2$ pour $4<d<6$ diffère de la valeur obtenue par la méthode de Flory $v_{\mathrm{F}}=3 / d$. On obtient également
\end{abstract}

$$
\gamma=1+\frac{3}{8} \varepsilon+\frac{15}{64} \varepsilon^{2}+0\left(\varepsilon^{3}\right) \text { où } \varepsilon=6-d .
$$

Une méthode directe du groupe de renormalisation par décimation le long de la séquence chimique permet également de déterminer $v=2 / d-2$ et de montrer que $v \equiv 1$ pour $d<4$.

Abstract. - The exponents $\gamma$ and $v$ describing the asymptotic properties of one polyelectrolyte chain in the limit of high molecular weight are determined using a zero component Lagrangian theory near $d=6$ dimensions. We find a polyion size $R \sim N^{2 / d-2}$ where $N$ is the polymerisation index. This result $v=2 / d-2$ for $4<d<6$ differs from the Flory-type result $v_{\mathrm{F}}=3 / d$. The exponent $\gamma$ related to the bahaviour of the internal partition function of one chain is obtained as an expansion in $\varepsilon=6-d$ to order $\varepsilon^{2}$ :

$$
\gamma=1+\frac{3}{8} \varepsilon+\frac{15}{64} \varepsilon^{2}
$$

A direct renormalization group method of decimation along the chemical sequence is also used to recover the result for $v$ and to show that $v \equiv 1$ for $d<4$.

It is well known that linear polyelectrolytes have a larger size than neutral polymers [1], and behave in a quite different way because of the long range Coulomb interactions between monomers. Recently some of us have presented qualitative remarks on the conformation of polyelectrolytes in 3 dimensions [2]. We give here a more precise discussion for a single chain and for an arbitrary dimensionality $d$.

We consider a chain of $N$ monomers where each monomer carries a charge $e$ (and to each charge $e$ corresponds a counterion $-e$ in the solution). We suppose that the only interaction between monomers is the Coulomb interaction

$$
U=\frac{e_{1} e_{2}}{\varepsilon_{0} R_{12}}
$$

(*) Laboratoire associé au C.N.R.S. where $\varepsilon_{0}$ is the dielectric constant of water (the short range excluded volume repulsion is neglected in comparison to the Coulomb repulsion). In the case of one single chain considered here, we shall completely neglect the effect of counterions : for $d=3$ this is correct provided that the charge is below a certain threshold corresponding to condensation [1]. For $d>3$ this is always expected to be correct.

Self-consistent calculations and Flory type theories [2] lead to an overall size of the polyion linear in $N$ for $d=3: R \sim N$. More generally, for a $d$ dimensional system these theories lead to the behaviour $R \sim N^{v}$ with $v_{\mathrm{F}}=3 / d$. This simple result is obtained crudely by minimizing the free energy

$$
F \sim \frac{R^{2} T}{N}+\frac{(N e)^{2}}{\varepsilon_{0} R^{d-2}}
$$


where the two terms correspond respectively to the elastic energy and the Coulomb energy $\left({ }^{1}\right)$.

The Lagrangian theory with $n=0$ components developed by J. des Cloizeaux [3] and applied to the study of neutral polymers, can be extended to the case of charged polymers. We then consider a function $\varphi_{j}(x)$ with $n$ components $(j=1, \ldots, n)$ defined in a $d$-dimensional space and we associate with these variables the probability law

$$
P(\varphi)=\exp \left(-A_{0}(\varphi)-A_{1}(\varphi)\right)
$$

with

$$
\begin{aligned}
& A_{0}(\varphi)=\frac{1}{2} \sum_{j} \int \mathrm{d}^{d_{x}}\left[\left(\nabla \varphi_{j}(x)\right)^{2}+r_{0} \varphi_{j}^{2}(x)\right] \\
& A_{1}(\varphi)=\sum_{j l} \int \mathrm{d}^{d_{x}} \int \mathrm{d}^{d_{y}} \frac{U}{|x-y|^{d-2}} \varphi_{j}^{2}(x) \varphi_{l}^{2}(y)
\end{aligned}
$$

where

$$
U=\frac{e^{2} C}{\varepsilon_{0} S(d)} .
$$

When $n$ is fixed to zero, the probability law applies directly to the problem of one isolated polyelectrolyte chain with the usual equivalence :

$$
G_{n \rightarrow 0}^{(2)}(\mathbf{k})=\sum_{N} \mathrm{e}^{-r_{0} N} Z_{N}(\mathbf{k})
$$

where $G^{(2)}(\mathbf{k})$ is the Fourier transform of the twopoint correlation function in the Lagrangian theory and $Z_{N}(\mathbf{k})$ is the Fourier transform of $Z_{N}(\mathbf{R})$, the number of chains of $N$ monomers between two points separated by $\mathbf{R}$.

Following S. Ma [4] the action $A(\varphi)=A_{0}(\varphi)+A_{1}(\varphi)$ can be written

$$
\begin{aligned}
A(\varphi)=\sum_{\mathbf{k}}\left\{\frac{1}{2}\left(r_{0}+k^{2}\right)\left(\sum_{j=1}^{n} \varphi_{j}(\mathbf{k}) \varphi_{j}(-\mathbf{k})\right)+\right. \\
+\frac{k^{2}}{2} C(\mathbf{k}) C(-\mathbf{k})+ \\
\left.+u_{0} C(\mathbf{k}) \sum_{\mathbf{p}} \sum_{j=1}^{n} \varphi_{j}(\mathbf{p}) \varphi_{j}(\mathbf{p}+\mathbf{k})\right\}
\end{aligned}
$$

with

$$
u_{0}=\sqrt{2} i U^{1 / 2}, \quad i=\sqrt{-1}
$$

( $\left.{ }^{1}\right)$ The laws of electrostatics have been generalized here to $d$ dimensions by keeping the Laplace equation in the absence of charges $\Delta \phi(\mathbf{r})=0$ and by generalizing the Poisson equation $\Delta \phi(\mathbf{r})=-C \rho(\mathbf{r})$ where $\rho(\mathbf{r})$ is the density of charges. For two particles of charge $e$ at a distance $R$, the potential is then written

$$
V(R)=\frac{C}{S(d)} e^{2} / R^{d-2}
$$

where $S(d)$ is the area of a $d$ dimensional hypersphere of radius unity. where a static Coulomb field $C(\mathbf{k})$ is introduced and where the terms in $A(\varphi)$ involving $C(\mathbf{k})$ are introduced to reproduce the Coulomb interaction

$$
\begin{array}{r}
U \sum_{\mathbf{k}_{1}, \mathbf{k}_{2}, \mathbf{k}_{3}, \mathbf{k}_{4}} \sum_{j, l} \frac{\phi_{j}\left(k_{1}\right) \phi_{j}\left(k_{2}\right) \phi_{l}\left(k_{3}\right) \phi_{l}\left(k_{4}\right)}{\left(k_{1}+k_{2}\right)^{2}} \times \\
\times \delta\left(\mathbf{k}_{1}+\mathbf{k}_{2}+\mathbf{k}_{3}+\mathbf{k}_{4}\right) .
\end{array}
$$

The $n=0$ Lagrangian theory has been worked out both from eq. (3) and from (4) using the renormalization group and the $\varepsilon=6-d$ expansion; details will be given elsewhere $[5,6]$. We get the following results for the exponents $v$ and $\eta$ to order $\varepsilon^{2}$.

$$
\begin{aligned}
& \frac{1}{v}=2-\frac{\varepsilon}{2}+0\left(\varepsilon^{3}\right) \\
& \eta=-\frac{\varepsilon}{4}-\frac{9}{32} \varepsilon^{2}+0\left(\varepsilon^{3}\right)
\end{aligned}
$$

and from Fisher's scaling law

$$
\gamma=(2-\eta) v=1+\frac{3}{8} \varepsilon+\frac{15}{64} \varepsilon^{2}+0\left(\varepsilon^{3}\right) .
$$

The calculations have not been extended to higher order in $\varepsilon$, but we expect the result $v^{-1}=2-\varepsilon / 2$ to hold to all orders in $\varepsilon$. It can then be rewritten

$$
v=\frac{2}{d-2} \text {. }
$$

This result is certainly correct for $d<6$ (for $d>6$ the classical result $v=1 / 2$ is expected). The $\varepsilon$ expansion can not tell one the limits of validity. The stability of the non-trivial fixed point $u^{*} \neq 0$ is given through the dimension $\lambda_{u}$ of $u$. We find [5]

$$
\lambda_{u}=-\varepsilon+\frac{67}{48} \varepsilon^{2}+0\left(\varepsilon^{3}\right)
$$

(for comparison, in the neutral polymer problem where $u$ is the excluded volume repulsion,

$$
\lambda_{u}=-\varepsilon+\frac{21}{32} \varepsilon^{2} .
$$

From the physics of the problem, $v$ cannot exceed 1 (the chain cannot be more than fully extended). From (7), $v$ reaches 1 when $d=4$. For $d$ less than 4 we expect that the polymer has a new rigid behaviour with $v \equiv 1$ (in the same manner phase transitions reach a new regime when $T_{\mathrm{c}}=0$ ). This statement will be discussed later in this note using a direct renormalization group method.

The result (7) can be recovered in a simple way. When $n=0$ the Coulomb field $C(\mathbf{k})$ remains a free field $\left(\langle C(\mathbf{x}) C(0)\rangle \sim \frac{1}{|\mathbf{x}|^{d-2}}\right)$. Furthermore, if a non-trivial $\left(u^{*} \neq 0\right)$ stable fixed point exists, then in the vicinity of this fixed point, from eq. (3), $r$ (the 
variable conjugate to the energy density) and $C(\mathbf{x})$ scale with the same dimension in the change of scale of the renormalization group transformation. The dimension of $r$ is equal to $1 / v$ and the dimension of the free field $C(\mathbf{x})$ is the normal dimension equal to $d-2 / 2$. This leads immediately to eq. (7) [7].

The value for $v$ (eq. 7) can also be recovered by a direct renormalization group method of decimation. $g$ monomers are put together to form a new monomer. At the $n$th step of this iteration procedure the length of the monomer is $a_{n}$ and the charge $Q_{n}$. The equations which describe the renormalization group transformation from step $n$ to $n+1$ are written

$$
\begin{aligned}
a_{n+1} & =g^{1 / 2} a_{n}(1+k) \\
Q_{n+1} & =g Q_{n}
\end{aligned}
$$

where the correction function $k$ is a function of $g$ and of the reduced coupling parameter

$$
u_{n}=\frac{Q_{n}^{2}}{T a_{n}^{d-2}} .
$$

From perturbation expansion $k=k\left(u_{n} g^{\varepsilon / 2}\right)$ where $\varepsilon=6-d$. No correction function is implied in the renormalization of the charge (this is equivalent to the fact that the Coulomb field remains free in the Lagrangian theory when $n \rightarrow 0$ ). From (9) we get

$$
\frac{u_{n+1}}{u_{n}}=g^{\varepsilon / 2}(1+k)^{-(d-2)} \text {. }
$$

The behaviour described by (10) is valid for $u$ sufficiently small. On the contrary when $u$ is large, the chain behaves as a rigid chain $a_{n+1} \sim g a_{n}+$ corrections and

$$
\frac{u_{n+1}}{u_{n}} \sim g^{4-d}+\text { corrections }
$$

Thus for $d>6$ the gaussian fixed point $u^{*}=0$ is stable. For $4<d<6$ there exists a non-trivial stable fixed point $u^{*} \neq 0$ (for $4<d<6, u_{n+1} / u_{n}$ being larger than 1 for $u_{n}$ small and less than 1 for $u_{n}$ large has to be equal to 1 when $u_{n}=u^{*}$ where $u^{*}$ is a stable fixed point) ; from (10) when $u_{n} \rightarrow u^{*}$,

$$
1+k\left(u^{*}, g\right)=g^{\varepsilon / 2(d-2)}
$$

and close to the fixed point $a_{n+1} \sim g^{v} a_{n}$ with

$$
\begin{array}{r}
v=\lim \frac{\log \frac{a_{n+1}}{a_{n}}}{\log g}=\frac{1}{2}+\frac{\log \left(1+k\left(u^{*}\right)\right)}{\log g}= \\
=\frac{2}{d-2} .
\end{array}
$$

When $d$ moves towards $d=4$, the stable fixed point moves towards infinity and stays at infinity for $d<4$ (no stable fixed point exists). This corresponds to the new rigid regime with $v \equiv 1$.

This note is concerned with new results for the conformation of one polyelectrolyte chain in a $d$-dimensional space. For $4<d<6$ some exact results are obtained for the exponent $v$ which show that the Flory type arguments lead to wrong results. The situation at $d=4$ and for $d<4(v \equiv 1)$ needs further quantitative analysis. The effect of the counterions and the case of several chains will be considered in the near future.

Acknowledgements. - One of us (P. P.) appreciated stimulating discussions with $\mathrm{T}$. Lubensky during the course of this study.

\section{References}

[1] Oosawa, F., Polyelectrolytes M. Dekker, N. Y. (1971).

[2] De Gennes, P. G., Pincus, P., Velasco, R. M., Brochard, F., $J$. Physique, to be published.

[3] Des Cloizeaux, J., J. Physique Colloq. 37 (1976) C1-255.

[4] Ma, S., Phys. Rev. Lett. 29 (1972) 1331.

[5] Pfeuty, P. and Velasco, R. M., to be published.
[6] The general $n$ component case has been studied recently, Lubensky, T. and Pfeuty, P., to be published.

[7] For the neutral polymer chain with excluded volume, the same formulation can be introduced, but a mass term would appear in eq. (3) for the Coulomb field; no fixed point can be reached and arguments which could lead to $v=2 / d$ can no be pursued. 